\title{
Ocular ochronosis in alkaptonuria patients carrying mutations in the homogentisate 1,2-dioxygenase gene
}

\author{
Ute Felbor, Yvonne Mutsch, Franz Grehn, Clemens R Müller, Wolfram Kress
}

\begin{abstract}
Aims-To assess the involvement of the recently identified human homogentisate 1,2-dioxygenase gene (HGO) in alkaptonuria (AKU) in two unrelated patients with ochronosis of the conjunctiva, sclera, and cornea.

Methods-A mutation screen of the entire coding region of the HGO gene was performed using single stranded conformational analysis after polymerase chain reaction with oligonucleotide primers flanking all 14 exons of the HGO gene. Fragments showing aberrant mobility were directly sequenced.

Results-Two homozygous missense mutations, L25P and M368V, were identified, each of which leads to the replacement of a highly conserved amino acid in the HGO protein.

Conclusions-The authors describe a novel mutation, L25P, in the German population and bring to 18 the total number of known HGO mutations.

(Br F Ophthalmol 1999;83:680-683)
\end{abstract}

Alkaptonuria (AKU; OMIM 203500) has played a paradigmatic role in the history of human and biochemical genetics. It was this rare autosomal recessive disorder that led Sir Archibald Garrod to demonstrate the applicability of the rediscovered Mendelian laws to Homo sapiens in $1902^{1}$ and to formulate the fundamental concept of "inborn errors of metabolism" in $1908 .^{2}$ Half a century later, La $\mathrm{Du}$ et al presented the first experimental evidence for a specific enzyme defect in humans: the deficiency of homogentisic acid 1,2-dioxygenase activity (HGO; EC No

Augenklinik,

Universität Würzburg, Würzburg, Germany

U Felbor

Y Mutsch

F Grehn

Institut für

Humangenetik,

Universität Würzburg,

Würzburg, Germany

C R Müller

W Kress

Correspondence to:

Wolfram Kress, Institut für

Humangenetik, Biozentrum,

Am Hubland, D-97074

Würzburg, Germany.

Accepted for publication 6 January 1999
1996 and 1997, the human HGO gene was finally cloned ${ }^{7}$ and characterised. ${ }^{10}$ Two missense mutations cosegregating with alkaptonuria in two Spanish pedigrees established HGO as the defective gene in AKU. ${ }^{7}$ The identification of a third missense and a frame shift mutation in Slovakian families further confirmed the role of HGO mutations in the pathogenesis of AKU. ${ }^{11}$ In the meantime, 13 additional mutations were found in unrelated alkaptonuric subjects from six European countries, Algeria, Turkey, ${ }^{12}$ and Japan. ${ }^{13}$ The present study describes the identification of two homozygous missense mutations in two unrelated elderly German patients who were first diagnosed with this congenital disorder only after their referral to ophthalmologists.

\section{Patients and methods}

Two patients were diagnosed as having AKU. Patient $\mathrm{H}$, a 71 year old woman, was transferred for assessment of choroidal melanoma, and patient L, a 69 year old woman, was diagnosed incidentally during hospitalisation for subretinal surgery of a haemorrhagic maculopathy in age related macular degeneration. Despite striking features of ochronosis, the diagnosis of alkaptonuria had never been established in either individual. For several generations, the families of both patients have been settled in two Bavarian villages located approximately 70 kilometres apart. The parents of both patients were dead and consanguinity was apparently absent from both families. Patient L reported five sibs and three children without symptoms of alkaptonuria. The only other affected family member was $P$, a 76 year old brother of patient $\mathrm{H}$ who was not examined by the authors. After informed consent for clinical and molecular genetic assessment was obtained, an ophthalmological examination, photodocumentation, urine oxidation test, and gas chromatographic and mass spectrometric determinations of homogentisic acid in urine and blood serum were performed.

For mutation analysis, $10 \mathrm{ml}$ EDTA blood samples were obtained from the two individuals as well as the affected brother, P. Genomic DNA was isolated from leucocyte nuclei according to standard non-organic extraction procedures. Based on the previously published genomic structure of the HGO gene, polymerase chain reaction (PCR) primers were designed flanking all 14 coding exons. ${ }^{11}$ PCR products were screened for sequence differences by single stranded conformational analysis (SSCA). ${ }^{14}$ described. ${ }^{8}$ Subsequently, the first gene encoding an HGO enzyme was cloned from the ascomycete fungus Aspergillus nidulans. ${ }^{9}$ In 


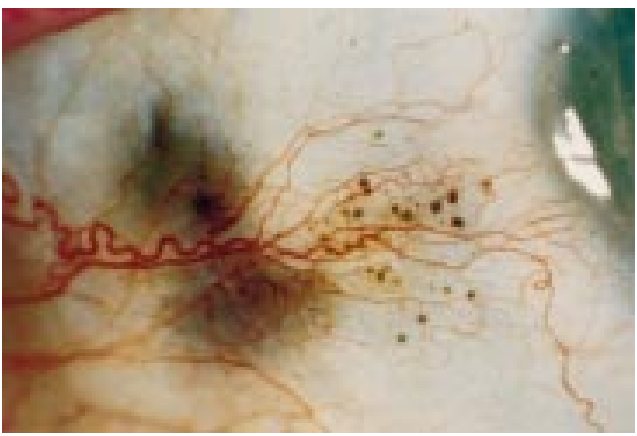

Figure 1 Ochronotic pigment in the conjunctiva, sclera, and peripheral cornea of 71 year old patient, $H$.

Aberrant fragments were directly sequenced using the Sequenase PCR product sequencing kit (United States Biochemical, Cleveland, $\mathrm{OH}$, USA).

\section{Results}

Each patient reported staining of urine from early childhood with the urine actually darkening to blackness on standing. The two patients, as well as the affected brother, had undergone various orthopaedic operations as a result of degenerative joint disease. Although pigmentation of the cartilage had been noted, the diagnosis of alkaptonuria was never established. On ophthalmological examination, the patients revealed bilateral grey brown to blue black pigmentation of the nasal and temporal sclera, bilateral drop-shaped pigmentation of the bulbar conjunctiva, and bilateral amber globules in the limbic cornea (Fig 1). Discoloration of the ears and an irregular thickening of auricular cartilage were observed bilaterally in both patients. Gas chromatographic and mass spectrometric analyses confirmed the clinical diagnosis of alkaptonuria. Excessive excretion of HGA in urine $(>2000 \mathrm{mmol} / \mathrm{mol}$ Crea, normal: $<100 \mathrm{mmol} / \mathrm{mol}$ Crea) and increased concentrations of HGA in blood serum were detectable in the two individuals.

To analyse the $\mathrm{HGO}$ gene in the two German alkaptonuria patients, SSCA of the entire coding region of the HGO gene was performed. Homozygous aberrant mobility shifts were detected in exons 2 and 13 in patient $\mathrm{L}$ and $\mathrm{H}$, respectively (Fig $2 \mathrm{~A}, 3 \mathrm{~A}$ ). No other band shifts were found in the remaining gene fragments. Subsequently, sequencing of the aberrant exon 2 PCR product revealed a homozygous $\mathrm{T}$ to $\mathrm{C}$ transition in the second position of codon 25 changing a leucine residue to proline (Fig 2B). This novel mutation, designated L25P, was not observed in a German control group including more than 100 chromosomes. The homozygous mutation identified in patient $\mathrm{H}$ and her affected brother P turned out to be the methionine to valine substitution at codon 368 ,
A

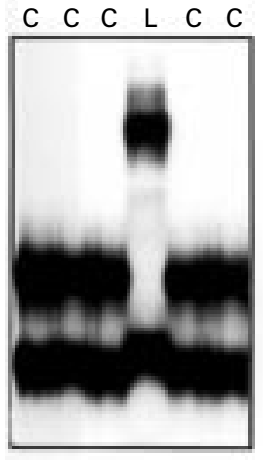

B

Figure 3 (A) SSCA of exon 13 of the HGO gene showed a homozygous band shift in patient $H(H)$. $(C)=$ controls, $(X)=$ unpublished heterozygous mutation. (B) Both patient $H$ and her affected brother $P(P)$ carry a homozygous $A$ to $G$ substitution changing codon 368 from methionine to valine $(\mathrm{M} 368 \mathrm{~V})$

Figure 2 Mutation analysis of exon 2 of the human $H G O$ gene. (A) SSCA revealed a homozygous mobility shift in patient $L(L)$ compared with controls $(C)$. (B) PCR sequencing detected a homozygous $T$ to $C$ transition at the second position of codon 25 changing a leucine residue to a proline (L25P).

A

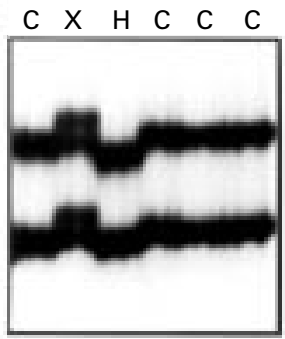

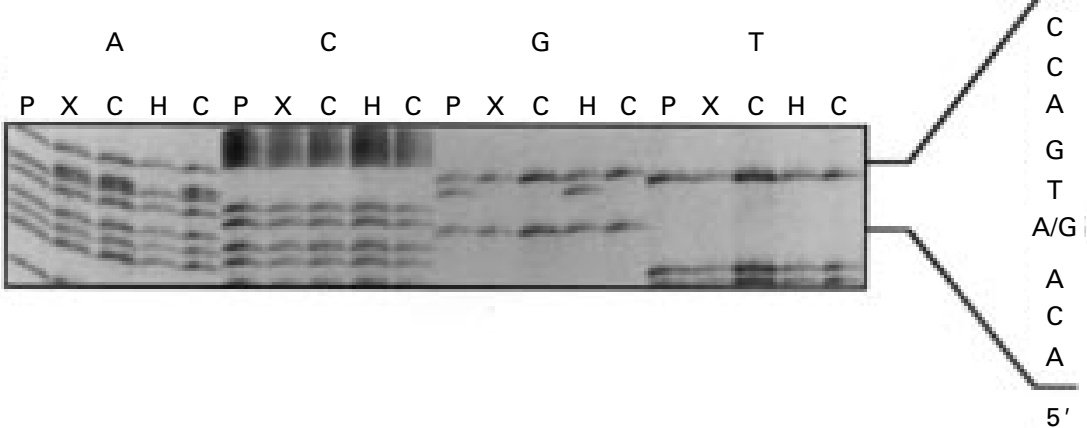

T 369

M368V 
M368V, previously identified in German subjects $^{12}$ (Fig 3B).

A comparison of the deduced amino acid sequences of the human, ${ }^{7}$ fungal ${ }^{9}$ (GenBank accession number U30797), and murine ${ }^{15}$ (U58988) HGO proteins demonstrates that both mutations lead to the replacement of amino acids that are conserved at the same relative locations between species which already indicates some functional significance. In addition, recombinant expression of the mutant $\mathrm{M} 368 \mathrm{~V}$ HGO protein in $E$ coli demonstrated loss of enzymatic activity. ${ }^{16}$

\section{Discussion}

In the present report, ocular ochronosis prompted the diagnosis of a congenital disorder, alkaptonuria, in two patients of about 70 years of age, one of whom was referred for evaluation of choroidal melanoma. It is not in fact surprising that ophthalmologists would encounter undiagnosed alkaptonuric patients. Involvement of the ocular tissue is widespread in AKU and was, for instance, found in all but one of 82 cases reviewed by Smith in $1942 .{ }^{17}$ Furthermore, ocular ochronosis usually precedes the appearance of articular symptoms and was the first manifestation of ochronosis in $70.5 \%$ of 119 Slovakian patients. ${ }^{18}$ In addition, the differential diagnosis of blue black scleral pigmentation includes malignant melanoma of the choroid and there is at least one tragic instance in which ochronotic pigment deposition was misdiagnosed as melanosarcoma and the patient's only eye was enucleated. ${ }^{19}$

$\mathrm{AKU}$ is a rare disorder with a prevalence of 1 in 250000 individuals. It is notably frequent in the Dominican Republic ${ }^{20}$ and Slovakia. ${ }^{21} \mathrm{~A}$ total of 191 alkaptonuria patients were registered at the Institute of Clinical Genetics in Martin, Slovakia, in $1994 .{ }^{6}$ Although founder effects and subsequent genetic isolation are known to cause high frequencies of relatively rare alleles in small populations, it is already evident that more than one founder must have contributed to the Slovakian gene pool. In addition to the previously published Slovakian frameshift and missense mutations, ${ }^{11}$ the P230S and V300G mutations, first identified in the Spanish population, ${ }^{7}$ have also been detected in Slovakian patients. ${ }^{16}$ Similarly, the occurrence of two different homozygous mutations in two valleys of a North Bavarian mountain range is likely to produce genetic isolates which can result in inbreeding and an increase in recessive disorders. Future mutational and haplotype analyses comparable with those performed in other isolated populations such as Finland would be required to clarify the origin, geographic distribution, and frequencies of the various AKU mutations.

In contrast with most other metabolic disorders, intellectual capacity and life expectancy are not reduced in AKU; however, ochronotic arthritis can be painful and disabling. Since ascorbic acid inhibits the oxidation of HGA to ochronotic pigment in vitro, its use has been suggested as a possible means of reducing pigment accumulation in connective tissue. ${ }^{22}$ Evidence supporting this was provided by alkap- tonuric mice which revealed homogentisic aciduria but showed no pigment deposits in knee and hip articular cartilage, intervertebral discs, tendons, and articular soft tissues. ${ }^{8}$ This lack of ochronosis in alkaptonuric mice was explained by the observed endogenous production of ascorbic acid in mice. ${ }^{23}$ However, there is no experimental proof that mice actually possess the enzyme system believed to mediate the conversion of HGA to its polymerised products in humans. In addition, the efficacy of ascorbic acid treatment has never been proved in a long term study of patients affected with alkaptonuria.

Recently, the triketone herbicide NTBC has been proposed as a potential treatment of AKU in combination with dietary restriction of phenylalanine and tyrosine. ${ }^{24}$ NTBC inhibits 4-hydroxyphenylpyruvate dioxygenase, the enzyme that produces homogentisic acid, and partially blocks the formation of oxidising tyrosine metabolites which cause severe liver cirrhosis, hepatocellular carcinoma, renal tubular dysfunction, and peripheral neuropathy in infants and children with tyrosinaemia type I. Its efficacy and safety are currently under detailed investigation in a group of patients affected with tyrosinaemia type $\mathrm{I}^{24}$ which results from deficiency in fumarylacetoacetate hydrolase, the last enzyme in the tyrosine catabolism pathway.

If pharmacotherapeutic approaches are not shown to successfully relieve the patients' symptoms, the identification of the AKU gene offers a new therapeutic strategy of enzyme replacement via recombinant production of HGO. However, it is presently not known whether the application of genetically engineered HGO protein would lead to an accumulation of reactive intermediates of the tyrosine catabolic pathway similar to that observed in tyrosinaemia type I. Therefore, genetic treatment needs to be carefully evaluated in animal models such as the aku mouse ${ }^{8}$ before human trials can be started. ${ }^{25}$ Nevertheless, the elucidation of the molecular basis of AKU has not only enabled reliable carrier detection in at risk populations but also a possible alternative therapeutic approach.

This study was supported by the Deutsche ForschungsgemeinThis study was supported by the Deutsche Forschungsgemeinschaft $(\mathrm{Kr} 1211 / 1-3)$. The
for technical assistance.

1 Garrod AE. The incidence of alkaptonuria: a study in clinical individuality. Lancet 1902;2:1616-20.

2 Garrod AE. The Croonian Lectures on inborn errors of Garrod AE. The Croonian Lectures on inborn errors of
metabolism. Lecture II. Alkaptonuria. Lancet 1908;2:73-9.

3 La Du BN, Zannoni VG, Laster L, et al. The nature of the $\mathrm{La}$ Du BN, Zannoni VG, Laster L, et al. The nature of the
defect in tyrosine metabolism in alcaptonuria. $\mathcal{F}$ Biol Chem defect in tyrosine

4 O'Brien WM, La Du BN, Bunim JJ. Biochemical, pathological and clinical aspects of alkaptonuria, ochronosis and ochronotic arthropathy: review of world literature (1584-1962). Am F Med 1963;34:813-38.

5 Pollak MR, Chou Y-HW, Cerda JJ, et al. Homozygosity mapping of the gene for alkaptonuria to chromosome $3 \mathrm{q} 2$. Nat Genet 1993;5:201-4.

6 Janocha S, Wolz W, Srsen S, et al. The human gene for alkaptonuria (AKU) maps to chromosome 3q. Genomics 1994; 19:5-8.

7 Fernández-Cañón JM, Granadino B, Beltrán-Valero de Bernabé $\mathrm{D}$, et al. The molecular basis of alkaptonuria. Nat Genet 1996;14:19-24.

8 Montagutelli X, Lalouette A, Coudé M, et al. aku, a mutation of the mouse homologous to human alkaptonuria, maps to chromosome 16. Genomics 1994;19:9-11. 
9 Fernández-Cañón JM, Peñalva MA. Fungal metabolic model for human type I hereditary tyrosinaemia. Proc Nat model for human type I hereditary
Acad Sci USA 1995;92:9132-6.

10 Granadino B, Beltrán-Valero de Bernabé D, FernándezCañón JM, et al. The human homogentisate 1,2 dioxygenase (HGO) gene. Genomics 1997;43:115-22

11 Gehrig, A, Schmidt SR, Müller CR, et al. Molecular defects in alkaptonuria. Cytogenet Cell Genet 1997;76:14-16.

12 Beltrán-Valero de Bernabé D, Granadino B, Chiarelli I, et al. Mutation and polymorphism analysis of the human homogentisate 1,2-dioxygenase gene in alkaptonuria patients. Am 7 Hum Genet 1998;62:776-84.

13 Higashino K, Liu W, Ohkawa T, et al. A novel point mutation associated with alkaptonuria. Clin Genet 1998;53: 228-9.

14 Orita M, Iwahana H, Kanazawa $\mathrm{H}$, et al. Detection of polymorphisms of human DNA by gel electrophoresis as single-stranded conformation polymorphisms. Proc Natl single-stranded conformation poly

15 Schmidt SR, Gehrig A, Koehler MR, et al. Cloning of the homogentisate 1,2-dioxygenase gene, the key enzyme of homogentisate 1,2-dioxygenase gene, the key enzyme
alkaptonuria in mouse. Mammal Genome 1997;8:168-71.

16 Müller CR, Fregin A, Srsen S, et al. Allelic heterogeneity of alkaptonuria patients in central Europe. (in press).
17 Smith JW. Ochronosis of the sclera and cornea complicating alkaptonuria. FAMA 1942;120:1282-8.

18 Cervenansky J, Sitaj S, Urbanek T. Alkaptonuria and ochronosis. F Bone ft Sur 1959;41-A:1169-82.

19 Skinsnes OK. Generalized ochronosis. Report of an instance in which it was misdiagnosed as melanosarcoma, with resultant enucleation of an eye. Arch Path 1948;45:552-8.

20 Milch RA. Studies of alcaptonuria: inheritance of 47 cases in eight highly inter-related Dominican kindreds. $A m$ f Hum Genet 1960;12:76-85.

21 Srsen S, Cisarik F, Pasztor L, et al. Alkaptonuria in the Trencin district of Czechoslovakia. Am f Med Genet 1978; 2:159-66.

22 Zannoni VG, Lomtevas V, Goldfinger S. Oxidation of homogentisic acid to ochronotic pigment in connective tissue. Biochim Biophys Acta 1969;177:94-105.

23 Levine $M$. New concepts in the biology and biochemistry of ascorbic acid. N Engl F Med 1986;314:892-902.

24 Anikster Y, Nyhan WL, Gahl WA. NTBC and alkaptonuria. Am $\mathcal{F}$ Hum Genet 1998;63:920-1.

$25 \mathrm{La} \mathrm{Du} \mathrm{BN}$. Are we ready to try to cure alkaptonuria? $A m \mathrm{f}$ Hum Genet 1998;62:765-7. 